Originally published as:

Kohse, K.P., Thamm, M.

KiGGS-the German survey on children's health as data base for reference intervals (2011) Clinical Biochemistry, 44 (7), p. 479.

DOI: 10.1016/j.clinbiochem.2011.02.016

This is an author manuscript.

The definitive version is available at: http://www.sciencedirect.com 


\title{
KiGGS-the German survey on children's health as data base for reference intervals
}

\author{
Klaus P. Kohse ${ }^{a}$, Michael Thamm ${ }^{b}$

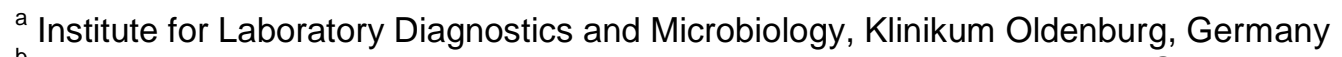 \\ ${ }^{b}$ Dept. Epidemiology and Health Reporting, Robert Koch-Institute, Berlin, Germany
}

\section{Introduction}

For a valid interpretation of the results obtained by laboratory investigations in children, the availability of age specific reference intervals is indispensible since the different phases of physiological development of the healthy child from or even before birth to adolescence are reflected in rather different concentrations of a variety of clinical laboratory analytes in blood. Numerous attempts have been made in the past to establish valid reference intervals in paediatric populations, but many of them were either limited in the number of children studied or by the use of methods which could not be regarded as universally standardized. In recent years, regional activities for the establishment of reference intervals have been performed with a substantial number of healthy children in each age group and well-characterized or traceable methods, such as the NORICHILD initiative of the Scandinavian Societies of Clinical Chemistry, or the CALIPER initiative carried out in Canada. In the United States, a long-term project, the National Children's Study, has been started a few years ago and will eventually yield clinical laboratory results on more than 100,000 children. Thanks to the German Health Interview and Examination Survey for Children and Adolescents (KiGGS), paediatric laboratories in Germany will be able to rely on a set of reference intervals for many parameters used in routine laboratory investigations based on a large number of children with well characterized health status.

\section{Methods}

In the KiGGS study [1] 167 study locations (sample points) were chosen all over Germany, and the subjects were randomly selected from the official registers of local residents. For the thorough evaluation of the health status, questionnaires were filled in by parents and (using parallel questionnaires) by children aged 11 years and older. The study team of physicians and health workers performed physical examinations and other tests as well as computer assisted personal interviews. About 18,000 children from age 0 to 18 years were studied, with a number of 1000 per age group comprising 1 year. Ca. 14,000 EDTA blood samples, ca. 14,000 serum samples and ca. 14,000 urine samples were collected from children older than 1 year. In younger children, the design of the study did not permit collection of blood and urine samples. Following a strict preanalytical protocol, the laboratory investigations were carried out at central laboratories using well-defined standardized methods (e.g., IFCC reference methods) when available [2].

\section{Results and discussion}

Areas of interest for the laboratory parameters investigated in the KiGGS study were general health indices (such as clinical chemistry analytes, red blood count, and urine status), markers of the nutritional state, the thyroid as well as the iron metabolism, indices for atopic sensitization (allergyspecific $\operatorname{lgE}$ ), and markers of past infections, or the immunization status. After the first evaluation of the data, a comprehensive overview of results for ca. 25 analytes of the study has recently been published [3]. It contains age dependent percentiles (3rd, 5th, 10th, 25th, 50th, 75th, 90th, 95th, and 97 th) in table as well as graphic form which were obtained using elaborate statistical methods in order to deal with nonparametric distributions. These data may already serve as a basis for age specific reference intervals, although it may still be necessary to examine the clinical data of the children studied and maybe rule out some of the results. On the other hand, since a variety of clinical 
information is available for these children, it may be possible to study the influence of parameters like body weight or other biometric and physiological data on some of the laboratory parameters investigated.

The data obtained during the KiGGS study are available from the Robert Koch-Institute as public use files for scientists with a documented interest in the field of paediatric medicine and after sending in a request containing the intended use of the data.

With the wealth of laboratory data obtained in the KiGGS study, it will now also be possible to compare the results of the laboratory investigations gathered in the other surveys, such as NORICHILD,

CALIPER, or the National Children's study.

\section{References}

[1] http://www.kiggs.de/service/english/index.html.

[2] W. Thierfelder, R. Dortschy, B. Hintzpeter, H. Kahl, C. Scheidt-Nave. Biochemical measures in the German Health Interview and Examination Survey for Children and Adolescents (KiGGS)

Bundesgesundheitsblatt Gesundheitsforschung Gesundheitsschutz 2007; 50: 757-770.

[3] R. Dortschy, A. Schaffarth Rosario, C. Scheidt-Nave, W. Thierfelder, M. Thamm, J. Gutsche, A. Markert. Bevölkerungsbezogene Verteilungswerte ausgewählter Laborparameter aus der Studie zur Gesundheit von Kindern und Jugendlichen in Deutschland (KiGGS). Beiträge zur

Gesundheitsberichterstattung des Bundes, in: L. Ryl (ed.) Robert Koch-Institut, Berlin 2009. 